\title{
Sensors for corrosion detection: measurement of copper ions in 3.5\% sodium chloride using screen-printed platinum electrodes
}

\author{
Andy Cranny, Nick R. Harris, Mengyan Nie, Julian A. Wharton, Robert J. K. Wood, and Keith R. Stokes
}

\begin{abstract}
Planar screen-printed platinum electrodes developed for use in corrosion monitoring have been evaluated using cyclic differential pulse voltammetry and shown to detect cupric ions $\left(\mathrm{Cu}^{2+}\right)$ over a range up to $100 \mathrm{mM}$ in a background of $3.5 \%$ by weight sodium chloride solution. The reduction of $\mathrm{Cu}^{2+}$ to metallic copper is shown to proceed as two successive singleelectron transfer reactions involving an intermediate chemical step where the cuprous ion $\left(\mathrm{Cu}^{+}\right)$is complexed by chloride to form the dichlorocuprous anion $\mathrm{CuCl}_{2}^{-}$. By comparison, the complexation step during the oxidation of copper to $\mathrm{Cu}^{2+}$ can involve a number of different chlorocopper(I) complexes of the general form $\left[\mathrm{CuCl}_{(\mathrm{n}+1)}\right]^{\mathrm{n}-}$ depending on the chloride concentration, which can make detection via a stripping reaction difficult.
\end{abstract}

Index Terms - Chemical sensor, copper, corrosion, thick film.

\section{INTRODUCTION}

$\mathrm{T}$ HE sea is a natural electrolyte which will ultimately initiate and promote the corrosion of many metals or alloys in contact with it. Prevention usually takes the form of the suitable deployment of sacrificial materials around a structure (e.g. cathodic protection) and/or the application of inhibitor coatings. Both of these methods whilst effective do however have a fixed lifetime and corrosion processes will in due course occur unless such remediation strategies are regularly updated. The corrosion of marine structures is of course not just detrimental from an economic perspective but can also have fatal consequences. Whilst structures such as ships can be removed from the marine environment for maintenance purposes, this is not an option with permanent installations such as oil platforms and pipelines. There is therefore an obvious requirement for in-situ corrosion monitoring to provide an early warning of its onset and avoid

Manuscript received April 19, 2011; revised November 28, 2011. This work was supported by the Engineering and Physical Sciences Research Council UK (EPSRC) and the Defence Science and Technology Laboratory (Dstl) under Grant EP/F004362/1.

A. Cranny and N. R. Harris are with the School of Electronics and Computer Science, University of Southampton, Hampshire, SO17 1BJ, UK (email: awc@ecs.soton.ac.uk; nrh@ecs.soton.ac.uk).

M. Nie, J. A. Wharton and R. J. K. Wood are with the National Centre for Advanced Tribology at Southampton, School of Engineering Sciences, University of Southampton, Hampshire, SO17 1BJ, UK (e-mail: m.nie@soton.ac.uk; j.a.wharton@soton.ac.uk; rjw3@soton.ac.uk).

K. R. Stokes is with the Physical Sciences Department, Defence Science and Technology Laboratory, Porton Down, Salisbury, Wiltshire, SP4 0JQ, UK (e-mail: krstokes@mail.dstl.gov.uk).

Digital Object Identifier 10.1109/JSEN.2012.2183867 potential structural failure and/or to help with the scheduling of service intervals.

Examples of materials used in the construction of marine based structures include various grades of steel as well as a number of copper-based alloys such as the copper nickels, aluminium bronzes and nickel-aluminium bronzes (NAB). Our group is particularly interested in the detection of copper ions released during the corrosion of $\mathrm{NAB}$ in marine environments since previous work by some of the authors has indicated that the detection of $\mathrm{Cu}^{2+}$ may serve as an early warning indicator of the onset of crevice corrosion: the anodic interfacial dissolution of copper occurring well before that of the minor nickel and iron alloying elements [1]. Copper ions can however be difficult to detect by conventional electrochemical methods due to their propensity to form complexes with chloride which, depending on the latter's concentration, can result in the formation of non-ionic compounds.

Examination of the available scientific literature reveals that there are a number of sensors being developed for copper ions using a variety of sensing techniques and covering a wide and disparate range of applications [2]-[11]. For example, an emerging trend reported in the literature is the development of potentiometric ion selective electrodes (ISE) particularly those utilising ionophores embedded in a polymer matrix [2]-[8]. These can be very simple to fabricate (e.g. by coating a platinum wire with the ion sensitive medium), yet still demonstrate good sensitivity, high selectivity and fast response [2]-[4]. The work reported by Firooz et al. is a good illustration of this type of sensor [2]. Developed for the determination of copper in milk powder samples, they describe a potentiometric copper(II) ISE based on a platinum wire coated with a PVC membrane containing a $\mathrm{Cu}^{2+}$ specific ionophore and report a linear response with a near-Nernstian slope of $28.2 \mathrm{mV}$ decade ${ }^{-1}$ over the $\mathrm{Cu}^{2+}$ ion concentration range $1 \mu \mathrm{M}$ to $100 \mathrm{mM}$, a detection limit of $0.5 \mu \mathrm{M}$ and operational lifetime of 2 months.

More traditional electrode structures using reference electrode systems enclosed within glass bodies containing an electrolyte and terminated at one end with an ion-selective membrane have also been explored [5]-[8]. An example of this is that reported by Ganjali et al. who used a silver-silver chloride $(\mathrm{Ag} / \mathrm{AgCl})$ electrode and $\mathrm{Cu}^{2+}$ ion-selective ionophore doped PVC membrane to produce a sensor with a 
sensitivity close to $30 \mathrm{mV}$ decade ${ }^{-1}$ over the concentration range $1 \mu \mathrm{M}$ to $100 \mathrm{mM}$, limit of detection of $0.5 \mu \mathrm{M}$, response time less than $15 \mathrm{~s}$ and lifetime of the order of 4 months [5].

The desire to mass produce potentiometric sensors at low cost has motivated many researchers to turn their attention to screen-printing techniques [9]-[11]. For instance, Koncki et al. produced a screen-printed copper(II) sensor based on $\mathrm{Cu}_{2} \mathrm{~S}$ immobilized in a polymer matrix and printed over a silver back contact [9]. They reported a linear response with a sensitivity of $30 \mathrm{mV}$ decade ${ }^{-1}$ over the $\mathrm{Cu}^{2+}$ concentration range of $20 \mu \mathrm{M}$ to $10 \mathrm{mM}$ with a lower limit of detection slightly better than $10 \mu \mathrm{M}$. They later modified their fabrication method and immobilized $\mathrm{Cu}_{2} \mathrm{~S}$ in a screenprintable graphite paste which demonstrated similar response over the $\mathrm{Cu}^{2+}$ concentration range of $10 \mu \mathrm{M}$ to $10 \mathrm{mM}$ with response time shorter than $10 \mathrm{~s}$ [10].

Although the various forms of potentiometric sensors described demonstrate reasonable levels of sensitivity to cupric ions, they also have some shortcomings that would not favor their use for corrosion detection in marine environments. For example, their limited lifetime (typically 2 to 6 months) would be a drawback in structural health monitoring applications where corrosion processes can occur over much longer time scales. Moreover, the use of copper ISEs in marine environments can prove challenging due to changes in sensitivity resulting from complexation of the cupric ion with chloride, present at high concentrations in the background electrolyte [12], [13]. In addition, naturally occurring organic matter and surfactants in seawater may interfere with the ISE response through adsorption and extraction processes. Ion-selective membrane type sensors and liquid filled glass body electrodes are also inherently fragile, restricting their use in challenging or aggressive environments where a robust sensor is required. Furthermore glass body electrodes generally require replenishment of the liquid electrolyte to maintain sensor response characteristics and therefore can not be considered to be maintenance free and inappropriate for applications requiring long term remote monitoring. The example devices described above are also limited in their operational $\mathrm{pH}$ range which would be detrimental in corrosion sensing where large changes in $\mathrm{pH}$ can occur, particularly in the crevice corrosion of marine structures where very acidic environments can evolve [14][17].

The development of ion sensors employing voltammetric techniques has also attracted some attention [18]-[20]. This type of sensor offers a number of additional benefits when compared to their potentiometric counterparts. Principle amongst these is that they are capable of detecting more than one electroactive species at the same time. In addition, they do not generally require additional chemistries or mechanisms to function (e.g. ionophores and membranes) and are therefore inherently simpler structures to fabricate. Indeed the simplest form of voltammetric sensor is a plain carbon electrode. For example Honeychurch et al. reported that an unmodified screen-printed carbon electrode in a background electrolyte of
$0.1 \mathrm{M}$ malonic acid can detect $\mathrm{Cu}^{2+}$ ions in water samples down to trace levels using cyclic voltammetry and anodic stripping differential pulse voltammetry with little or no interference on the response from a range of other ionic species [18].

An interesting $\mathrm{Cu}^{2+}$ ion sensor that negates the requirements for a reference electrode is the micro-electro-mechanical beam resonator described by Rahafrooz and Pourkamali [21]. Here, aqueous copper ions oxidize the surface of a micromachined silicon beam resulting in the deposition of a thin metallic layer with associated increase in beam mass and concomitant change to the resonant frequency, which can be measured as an indicator of the film thickness. Through calibration this can be directly related to the background ion concentration and a lower limit of detection for $\mathrm{Cu}^{2+}$ ions of $4 \mu \mathrm{M}$ is reported. The device however needs to be removed from solution, cleaned and dried before measurements of the resonant frequency can be made; a drawback for an autonomous corrosion detection system. It also has the disadvantage of not being ion specific: the beam can be oxidized by a range of different metal cations.

This current study has explored the development of voltammetric sensors for the detection of corrosion byproducts (metal cations) primarily from copper-nickel alloys used in marine environments. The nature of these environments and the area of application are such that robust sensors are required with the minimum of maintenance and capable of sustained operation over long time scales. As will be shown, unmodified screen-printed platinum electrodes could potentially fulfill these requirements.

\section{EXPERIMENTAL}

\section{A. Electrode Fabrication}

Screen-printed platinum electrodes were fabricated using standard thick-film processing techniques under clean-room conditions upon $100 \mathrm{~mm} \times 100 \mathrm{~mm} \times 1 \mathrm{~mm}$ thick alumina substrates (Hybrid Laser Tech) that had been laser scribed to allow individual sensors of size $10 \mathrm{~mm} \mathrm{x} 30 \mathrm{~mm}$ to be snapped out on completion. Each electrode involved the printing and processing of three separate layers through stainless steel mesh screens: a platinum conductor; an insulator to define the electrode area and a solderable termination. A single sensor consisted of a pair of electrodes with active areas of different sizes defining the counter and working electrodes, with the former being larger than the latter. The platinum paste used (Heraeus Silica and Metals, RP10001-145B) is not a conventional thick-film material in that it does not contain a glass binder. Instead, platinum is dispersed within an organic resin which is completely burnt off during the firing process producing metal films of high purity that are considerably thinner than normal thick-films, typically less than $1 \mu \mathrm{m}$.

Fig. 1 shows the arrangement of the individual layers of a single sensor consisting of a circular counter electrode of diameter $2.6 \mathrm{~mm}$ and circular working electrode of diameter 


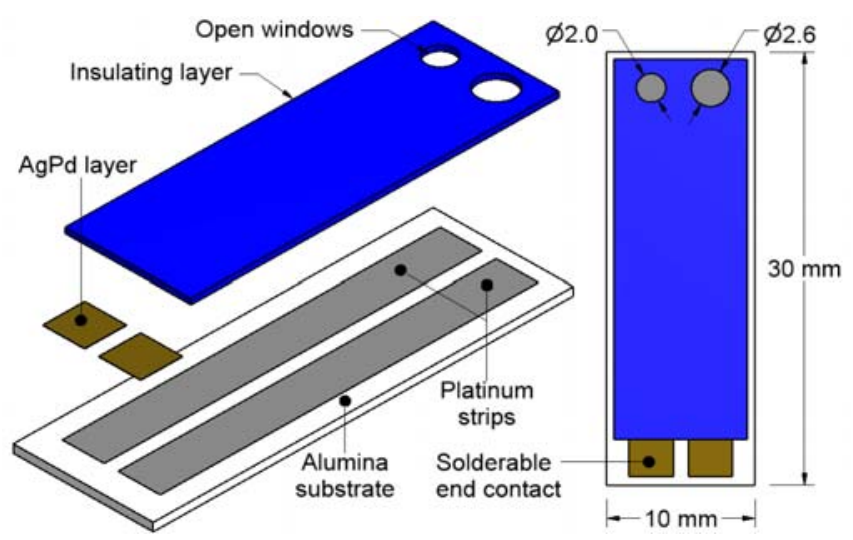

Fig 1. Schematic showing layers used in the fabrication of a single sensor.

$2.0 \mathrm{~mm}$. Each layer was printed sequentially using a DEK 248 screen printer. After printing each layer, the electrodes were left for 10 minutes to allow settling of the printed wet layer which removes any mesh imprints imposed on the film surface from the printing screen. Each layer was then dried in a 4zone belt furnace at a peak temperature of $150{ }^{\circ} \mathrm{C}$ over a 15 minute cycle (BTU conveyor furnace) and then fired in an 8zone belt furnace (BTU fast fire conveyor furnace) with peak temperature of $850^{\circ} \mathrm{C}$, temperature ascent and descent rates of $50{ }^{\circ} \mathrm{C} / \mathrm{min}$ and a total cycle length of $1 \mathrm{~h}$.

The metal electrodes were printed as $3 \mathrm{~mm}$ wide strips upon the alumina substrates and each was terminated at one end with a layer of silver-palladium paste (Electro Science Laboratories, 9635) to provide solderable electrical connection pads. A dielectric paste (Electro Science Laboratories, $4905 \mathrm{CH}$ ) was then printed over the majority of the metal electrodes, to an average thickness of $50 \mu \mathrm{m}$, to serve as an insulation layer and with windowed areas defining the active electrode geometries. To facilitate connection to the measurement instruments, a short length of a multi-core wire was soldered to the terminal ends of each electrode and sealed with room temperature vulcanizing rubber silicone (Dow Corning, 744). A photograph of a sensor is shown in Fig. 2.

\section{B. Solutions and Reagents}

In the experiments reported, the background electrolyte was an aqueous solution of $3.5 \%$ by weight sodium chloride produced by dissolving $\mathrm{NaCl}$ (VWR, AnalaR, >99.5\%) in freshly prepared de-ionized water (Elga Purelab Option, reverse osmosis water purification system, $>15 \mathrm{M} \Omega-\mathrm{cm})$. The chloride ion concentration of this solution is of the order 620 $\mathrm{mM}$ which is approximately equal to that of seawater at standard temperature and pressure [22]. A stock solution containing cupric $\left(\mathrm{Cu}^{2+}\right)$ ions at a concentration of $200 \mathrm{mM}$ was produced gravimetrically by dissolving copper(II) chloride dihydrate (Sigma Aldrich, ACS Reagent, 99+\%) in to a separate volume of $3.5 \%$ sodium chloride solution. Cupric ion concentration levels were varied during experiments by drop-wise addition of aliquots of this stock solution into a fixed volume of the background electrolyte using micropipettes. By preparing the $\mathrm{Cu}^{2+}$ stock solution in a $3.5 \%$ sodium chloride solution, the background chloride level was

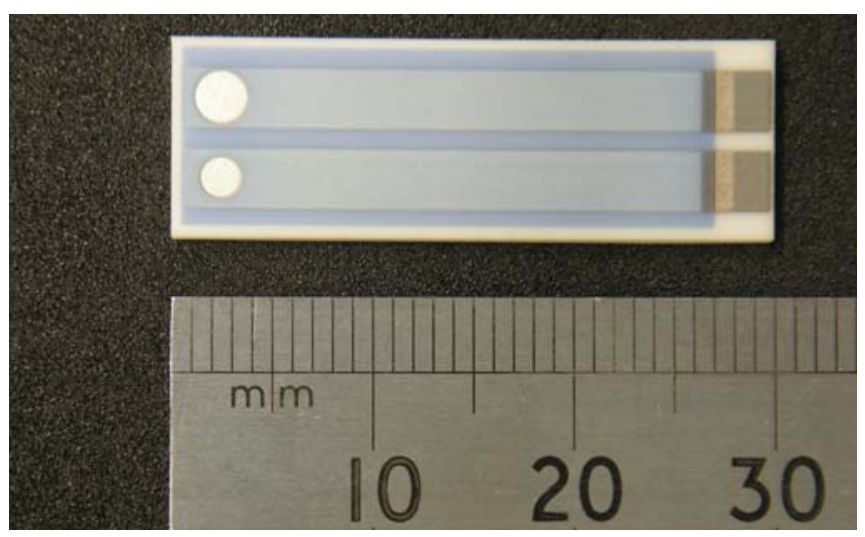

Fig. 2. Photograph showing a single screen-printed sensor.

not diluted; in fact a theoretical calculation based on the available mass of chloride and assuming full dissociation predicts that the chloride level increases from $620 \mathrm{mM}$ to 810 $\mathrm{mM}$ over the range of $\mathrm{Cu}^{2+}$ concentrations investigated. All reagents were used as supplied with no further treatment. Intentionally, no measures were taken to de-oxygenate the test solutions since the ability to de-gas sample solutions rarely presents itself in real world measurement applications.

\section{Instrumentation and Measurements}

All measurements were performed using an Autolab potentiostat (Pgstat302N) in standard 3-electrode arrangement with potential waveforms set with respect to an $\mathrm{Ag} / \mathrm{AgCl}$ reference electrode (VWR, GelPlas, 3.5 M KCl). The potential waveforms were generated using Nova 1.5 software (Eco Chemie) which was also used for signal recording. Experiments were undertaken in covered glass dishes which were thoroughly cleaned with de-ionized water between experiments. A magnetic stirrer was used to thoroughly mix solutions each time they were changed, but switched off during measurement periods. Temperature was not controlled, though it was noted that room temperature varied by no more than $\pm 1{ }^{\circ} \mathrm{C}$ about an average value of $24{ }^{\circ} \mathrm{C}$.

The electrodes were characterized using the electroanalytical technique of differential pulse voltammetry (DPV) where the working electrode current is measured prior to and at the end of a pulse superimposed on a staircase potential ramp, with the difference signal being recorded. DPV is generally accepted as being more sensitive than conventional cyclic voltammetry because it can discriminate against the large capacitive currents associated with the charging and discharging of the electrical double layer at the electrode surface as the potential of the electrode is rapidly changed during a voltammetric sweep. In addition, under the right conditions DPV is capable of distinguishing between $\mathrm{CE}, \mathrm{EC}$ and catalytic processes, where $\mathrm{C}$ represents a chemical step (in solution) and $\mathrm{E}$ an electrochemical step (at the electrode) [23].

In the investigation, the cupric concentration was varied over the range $0.1 \mathrm{mM}$ to $100 \mathrm{mM}$ in 20 arbitrary increments. This range was chosen since previous studies on the evolving crevice solution chemistries of corroding NAB revealed $\mathrm{Cu}^{2+}$ ion concentrations in the range $1.6 \mathrm{mM}$ to $80 \mathrm{mM} \mathrm{[1]} \mathrm{To}$ 
observe both reduction and oxidation reactions, DPV waveforms were programmed to scan potentials in both the cathodic and anodic directions. The relevant parameters for this cyclic DPV waveform were: step size $2 \mathrm{mV}$; interval time $100 \mathrm{~ms}$; pulse height $50 \mathrm{mV}$ and pulse length $40 \mathrm{~ms}$, giving an effective potential scan rate of $20 \mathrm{mV} / \mathrm{s}$. For each experiment two complete cycles were performed and the results presented correspond to the currents measured during the second cycle when equilibrium conditions are more likely to have been established. The $\mathrm{pH}$ of the solution was also measured for each cupric ion concentration using a ceramic junction glass bulb $\mathrm{pH}$ electrode (VWR, 662-1794) connected to a Hanna Instruments $211 \mathrm{pH}$ meter, which had been calibrated in $\mathrm{pH} 4$ and $\mathrm{pH} 7$ buffers before the start of the experiment.

Prior to performing experiments with cupric ions, the platinum electrodes were first characterized in just the background electrolyte primarily to establish the potential limits over which they could be operated without initiating the hydrolysis of water with the subsequent evolution of hydrogen and oxygen gases; the production of which would result in large electrode currents that would mask the signals of interest and greatly reduce sensitivity.

\section{RESULTS}

Fig. 3 shows cyclic DPV voltammograms obtained for screen-printed platinum electrodes in the background electrolyte recorded over the potential range $\pm 1000 \mathrm{mV}$ with the scan proceeding from $+1000 \mathrm{mV}$ down to $-1000 \mathrm{mV}$ and back to $+1000 \mathrm{mV}$. Fig. 3 reveals the presence of a single redox couple within the potential range $\pm 200 \mathrm{mV}$ which is attributed to the formation (oxidation) and stripping (reduction) of platinum oxide at the working electrode surface. As will be shown, however, the magnitudes of the currents at these peaks is significantly lower than those recorded during measurement at the lowest $\mathrm{Cu}^{2+}$ ion concentration investigated and therefore will not mask the sensor response.

Fig. 3 also shows that the platinum electrodes can be safely operated at anodic potentials up to $+1000 \mathrm{mV}$ without oxygen evolution occurring. However, the presence of large currents at potentials more negative than approximately $-800 \mathrm{mV}$ suggests that this potential sets the cathodic limit before hydrogen evolution commences.

\section{A. Full Potential Scan}

From the results of Fig. 3, the potential range $\pm 800 \mathrm{mV}$ was chosen to characterize the cupric response of the sensor, with scans proceeding in the direction $+800 \mathrm{mV}$ down to $-800 \mathrm{mV}$ then back to $+800 \mathrm{mV}$. Furthermore, for each concentration level investigated, the working electrode was cleaned after the potential scan by holding it at a potential of $+800 \mathrm{mV}$ for $30 \mathrm{~s}$.

Fig. 4 shows two series of example voltammograms recorded over six different low and six different high $\mathrm{Cu}^{2+}$ concentrations. The voltammograms show four distinct peaks in the current response: two reduction peaks during the forward scan (1 and 2) and two oxidation peaks during the

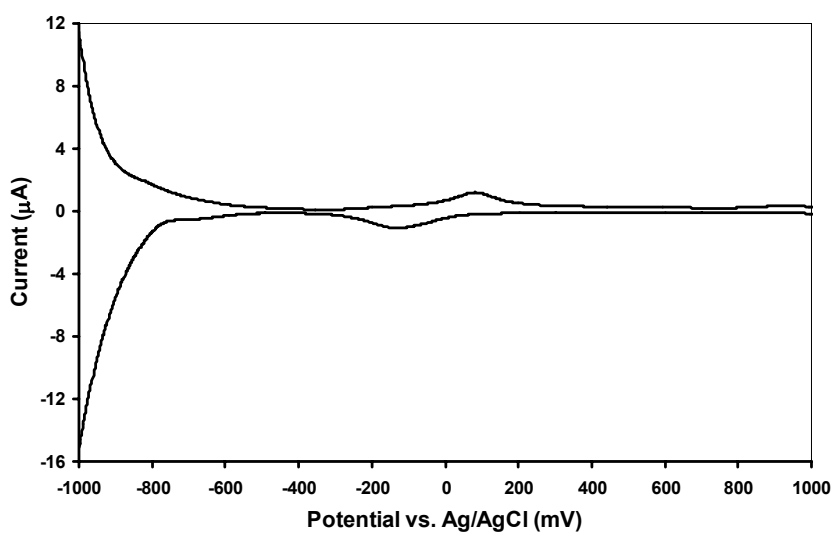

Fig. 3. Cyclic DPV waveform for platinum electrodes in $3.5 \% \mathrm{NaCl}$ solution.

reverse scan (3 and 4). For comparison, the inset in the left hand figure shows a voltammogram obtained with a platinum wire working electrode (diameter $0.5 \mathrm{~mm} \times 10 \mathrm{~mm}$ length) and screen-printed platinum counter electrode ( $3 \mathrm{~mm} \times 5 \mathrm{~mm}$ ) at a $\mathrm{Cu}^{2+}$ concentration of $20 \mathrm{mM}$ recorded using the same cyclic DPV parameters. This produces a very similar response with four current peaks located at similar potentials, suggesting that screen-printed platinum electrodes demonstrate an electrochemical response to $\mathrm{Cu}^{2+}$ that is comparable with that of pure metallic platinum and giving confidence in their use as electrochemical sensors.

The presence of four peaks implies the occurrence of two distinct redox processes. It has been reported that the current peaks correspond to the following reactions: the reduction of $\mathrm{Cu}^{2+}$ to $\mathrm{Cu}^{+}$in solution (Peak 1); the reduction (plating) of $\mathrm{Cu}^{+}$to metallic copper at the working electrode surface (Peak 2 ); the oxidation (stripping) of metallic copper from the working electrode to $\mathrm{Cu}^{+}$(Peak 3) and finally the oxidation of $\mathrm{Cu}^{+}$to $\mathrm{Cu}^{2+}$ in solution (Peak 4) [24]-[28]. During the investigation, a copper colored film was observed to develop over the surface of the working electrode during the formation of Peak 2 which was subsequently removed during the formation of Peak 3, confirming that these two current peaks do represent the plating and stripping of copper.

The example voltammograms shown in Fig. 4 have been selected to illustrate some general trends that are observed over the concentration range investigated. At low $\mathrm{Cu}^{2+}$ concentrations all four peaks are well defined with symmetric profiles and exhibit an increase in magnitude with increasing $\mathrm{Cu}^{2+}$ ion concentration. In addition, over these low $\mathrm{Cu}^{2+}$ concentrations the potentials at which Peaks 1 and 4 occur remain fairly constant whilst the potentials at which Peaks 2 and 3 occur shift toward more positive values with increasing concentration. By contrast, at higher $\mathrm{Cu}^{2+}$ concentrations the two oxidation peaks (Peak 3 and 4) become progressively broader with increasing $\mathrm{Cu}^{2+}$ concentration; most noticeably the stripping peak, which also becomes increasingly asymmetric in shape. This might be due to the superposition of a number of evolving current peaks (viz. electrode processes) confined within a narrow range of electrode potentials, suggesting that more than one electrode stripping 

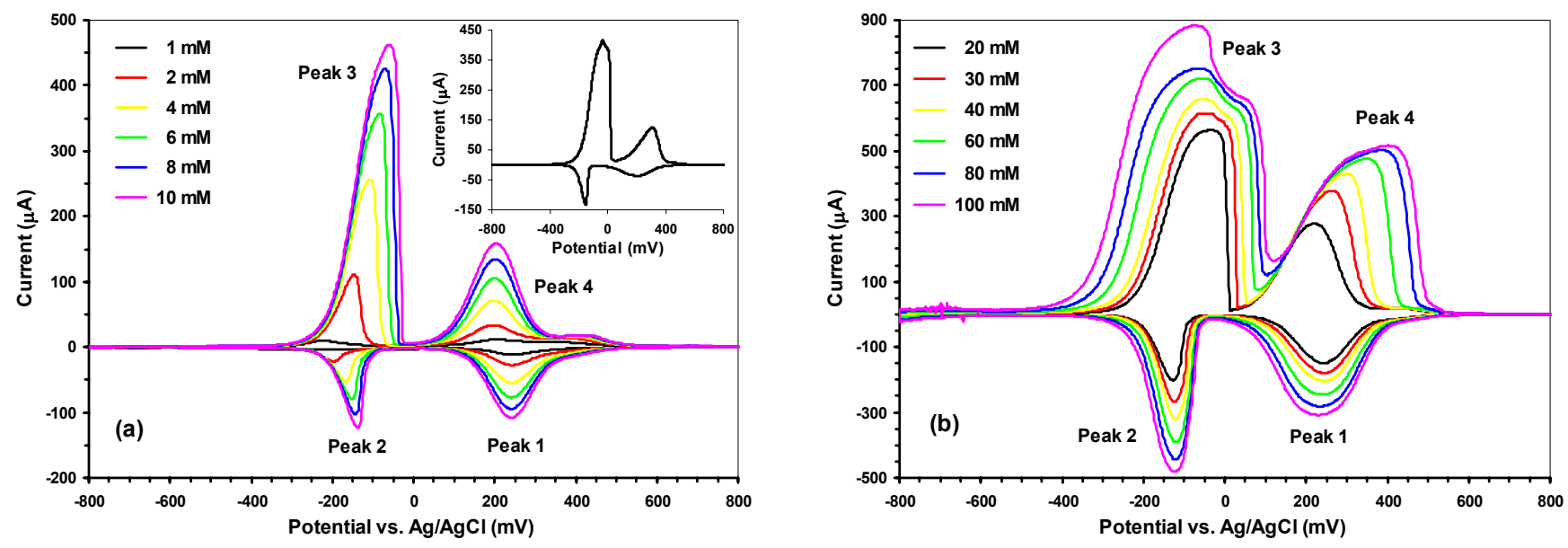

Fig. 4. Cyclic DPV voltammograms at selected low (a) and high (b) $\mathrm{Cu}^{2+}$ concentrations in $3.5 \% \mathrm{NaCl}$ for screen-printed platinum electrodes. Inset in left hand figure shows response of a platinum wire electrode to $20 \mathrm{mM} \mathrm{Cu}^{2+}$.

reaction could be involved at the higher $\mathrm{Cu}^{2+}$ concentrations. Fig. 4 also shows that whilst the potential at which the reduction of $\mathrm{Cu}^{2+}$ occurs remains fairly constant (Peak 1), the peak potential of Peak 4 (which was also fairly constant at low $\mathrm{Cu}^{2+}$ concentrations) now shifts dramatically toward more positive values with increasing concentration. These effects are better illustrated in Fig. 5 and Fig. 6 which respectively show the absolute values for peak current (i.e. without sign) and the potentials for each of the four peaks as a function of the $\mathrm{Cu}^{2+}$ concentration, where a secondary $\mathrm{x}$-axis indicating the corresponding chloride concentrations is also included for reference.

From the data presented in Fig. 5 it is clear that the relationships between peak current and $\mathrm{Cu}^{2+}$ concentration are not as expected if the previously described simple processes were solely responsible for each peak, where linear trends would result [29]. For example, the stripping peak (Peak 3) demonstrates a distinctly logarithmic characteristic, though as will be shown, this is a consequence of the summing of more than one stripping reaction occurring as the $\mathrm{Cu}^{2+}$ concentrations is varied, making it difficult to isolate the current contributions from individual reactions during data analysis and hence distorting the true trend.

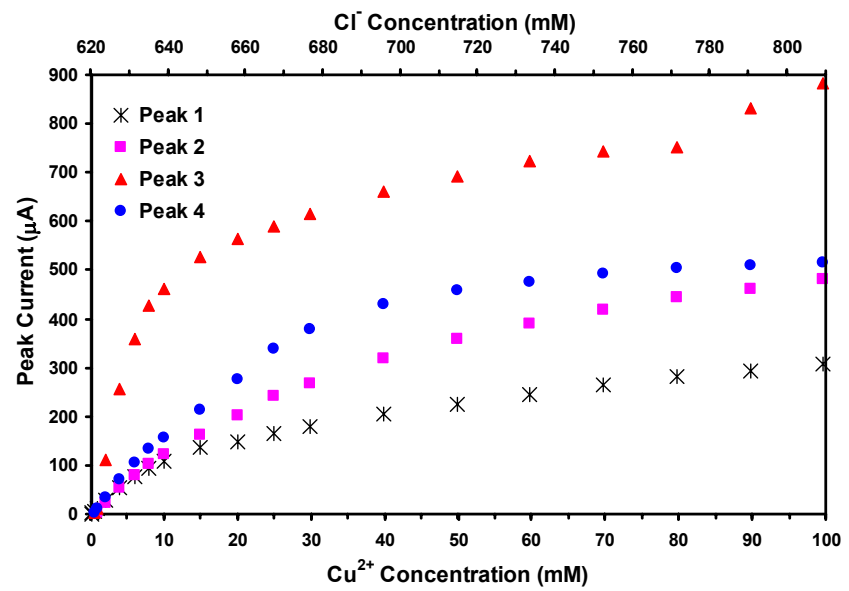

Fig. 5. Absolute peak current as a function of $\mathrm{Cu}^{2+}$ and $\mathrm{Cl}^{-}$concentrations.
Fig. 6 shows the variation in the peak potentials as a function of the $\mathrm{Cu}^{2+}$ concentration. Noticeable here is that the potential for the reduction of $\mathrm{Cu}^{2+}$ to $\mathrm{Cu}^{+}$(Peak 1) remains constant over the concentration range investigated, which agrees with Nernstian theory for a reaction where the concentrations of reactant and product are identical. The figure also shows that the potentials for the more cathodic redox couple (Peak 2 and 3) initially vary in a logarithmic manner for $\mathrm{Cu}^{2+}$ concentrations below approximately $20 \mathrm{mM}$ before leveling out at fairly constant values. The data for the second oxidation peak (Peak 4) is also quite revealing: demonstrating a shift in the position of the current peak to more anodic potentials as the $\mathrm{Cu}^{2+}$ concentration is increased before eventually settling at a stable level. The shift in potential (approximately $180 \mathrm{mV}$ ) is far greater than that predicted by the Nernst equation for a change in ion concentration of just two decades.

At the end of the experiment, a visual examination of both the working and counter electrodes revealed them to be intact and free of any surface deposits.

\section{B. Reduced Potential Scan}

A second experiment was performed in $3.5 \% \mathrm{NaCl}$ solution

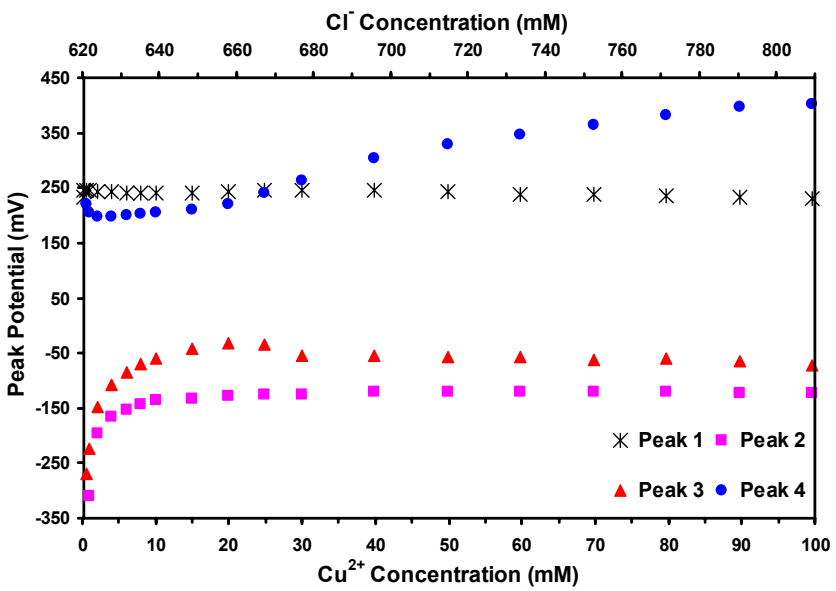

Fig. 6. Change in peak potential as a function of $\mathrm{Cu}^{2+}$ and $\mathrm{Cl}^{-}$concentrations. 


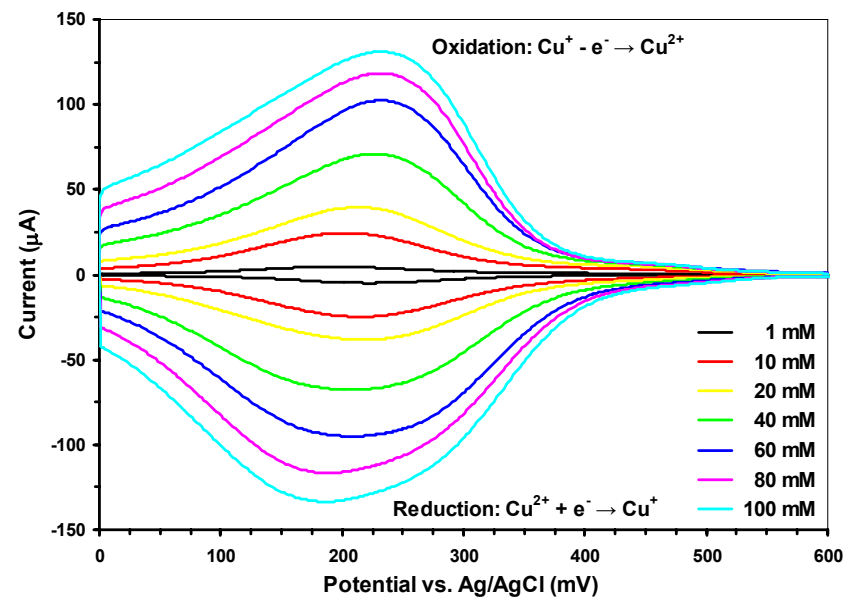

Fig. 7. Cyclic DPV voltammograms at selected $\mathrm{Cu}^{2+}$ concentrations in $3.5 \%$ $\mathrm{NaCl}$.

using a fresh pair of screen-printed platinum electrodes with the same DPV waveform parameters as previously described except that the potential range was reduced to between $0 \mathrm{mV}$ and $+600 \mathrm{mV}$ to avoid plating the working electrode with a metallic film at cathodic potentials. In this investigation, the potential scan proceeded from $+600 \mathrm{mV}$ down to $0 \mathrm{mV}$ then back to $+600 \mathrm{mV}$. Representative waveforms from this experiment are shown in Fig. 7 and reveal just two current peaks corresponding to the redox couple $\mathrm{Cu}^{2+} / \mathrm{Cu}^{+}$. By comparison with Fig. 4 it is noticeable that the potentials at which the oxidation peaks occur remain relatively constant over the $\mathrm{Cu}^{2+}$ concentration investigated.

Fig. 8 shows the variation in the absolute values for the peak currents as a function of the $\mathrm{Cu}^{2+}$ and $\mathrm{Cl}^{-}$concentration. This reveals a near linear relationship, with departures from linearity attributed to experimental variation (e.g. fluctuations in temperature). The data also shows that the values for current at each ion concentration are almost identical for both the oxidation and reduction processes, indicating a very reversible reaction.

A third experiment was performed with the same parameters as described above, but with $\mathrm{Cu}^{2+}$ ion concentrations varied over the much lower range of $10 \mu \mathrm{M}$ to $1000 \mu \mathrm{M}$ and with a sensor with slightly larger working electrode area of $4.0 \mathrm{~mm}^{2}$. Over this range, the calculated chloride concentration increases by only $2 \mathrm{mM}$ from an initial level of $621 \mathrm{mM}$. Similar voltammograms as those shown in Fig. 7 were observed. Fig. 9 shows the absolute values for the peak currents as a function of the $\mathrm{Cu}^{2+}$ concentration to which trend lines have been fitted. The results reveal that the sensor clearly responds to $\mathrm{Cu}^{2+}$ concentrations as low as $10 \mu \mathrm{M}$, and exhibits very linear responses both in reduction and oxidation for $\mathrm{Cu}^{2+}$ concentrations over the range $40 \mu \mathrm{M}$ to $1000 \mu \mathrm{M}$, with $\mathrm{R}^{2}$ correlations of the order of 0.98 .

\section{DISCUSSION}

\section{A. Electron Transfer Processes}

The characteristic 4-peak voltammograms displayed in Fig.

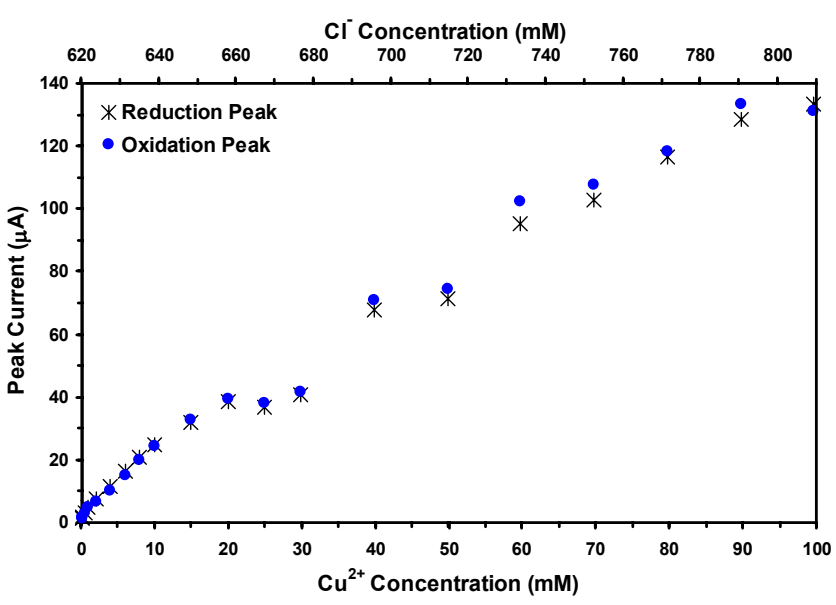

Fig. 8. Absolute peak current as a function of $\mathrm{Cu}^{2+}$ and $\mathrm{Cl}^{-}$concentration for $\mathrm{Cu}^{2+}$ concentrations over the range $0.1 \mathrm{mM}$ to $100 \mathrm{mM}$.

4 have also been reported by others using a variety of electrode materials and/or different voltammetric techniques in various chloride media [23]-[27]. In general the consensus is that the voltammograms represent two separate single electron transfer reactions. In the presence of high chloride backgrounds this is, however, an overly simplified explanation. Depending on the composition of the background electrolyte, the reduction of $\mathrm{Cu}^{2+}$ to metallic copper can proceed as a single two-electron transfer process given by:

$$
\mathrm{Cu}_{(\mathrm{aq})}^{2+}+2 \mathrm{e}^{-} \leftrightarrow \mathrm{Cu}_{(\mathrm{s})} \quad @+132 \mathrm{mV}
$$

Alternatively, the reduction may proceed as two successive single-electron reactions involving the cuprous ion $\left(\mathrm{Cu}^{+}\right)$as an intermediary given by:

$$
\mathrm{Cu}_{(\mathrm{aq})}^{2+}+\mathrm{e}^{-} \leftrightarrow \mathrm{Cu}_{(\mathrm{aq})}^{+} \quad \text { @ }-52 \mathrm{mV}
$$

followed by

$$
\mathrm{Cu}_{(\mathrm{aq})}^{+}+\mathrm{e}^{-} \leftrightarrow \mathrm{Cu}_{(\mathrm{s})} \quad @+316 \mathrm{mV}
$$

The potentials given in (1) to (3) are the standard reduction potentials with respect to an $\mathrm{Ag} / \mathrm{AgCl}(3.5 \mathrm{M} \mathrm{KCl})$ reference electrode and for $1 \mathrm{M}$ concentration of the ion species at a temperature of $25{ }^{\circ} \mathrm{C}$. The presence of four peaks in the recorded voltammograms does suggest a two-stage process

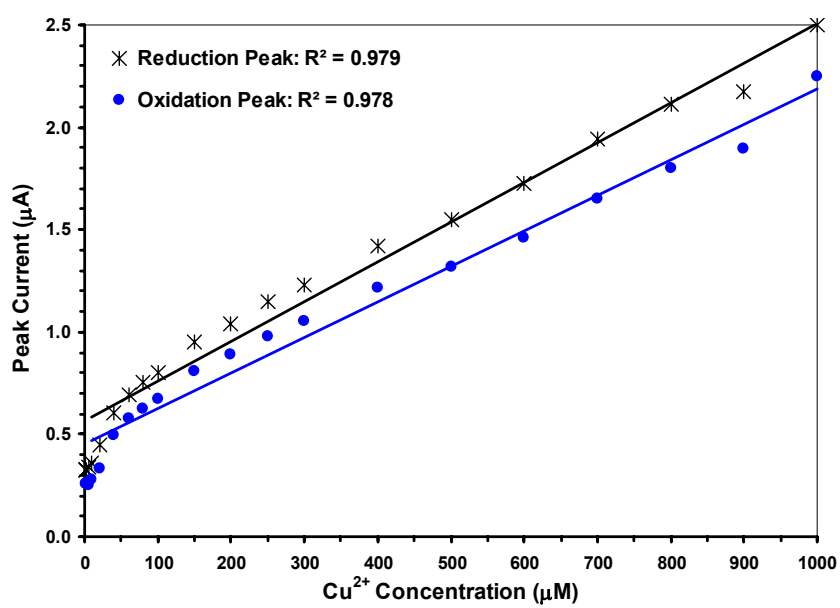

Fig. 9. Peak current as a function of $\mathrm{Cu}^{2+}$ over the range $10 \mu \mathrm{M}$ to $1000 \mu \mathrm{M}$. 
though the potentials at which the observed current peaks occur when corrected for concentration do not agree with those given by (2) and (3). Furthermore, given that the reaction described by (2) must precede that given by (3), the observation that the two reduction peaks shown in Fig. 4 occur sequentially as the electrode voltage is scanned in a negative direction (i.e. the potentials of the peaks descend numerically in value) is also inconsistent with the order predicted by (2) and (3) which shows that the potentials ascend in value. This apparent ambiguity can nevertheless be explained when we consider the affect that the chloride ion has on the electrochemical reactions.

The cuprous ion is not particularly stable in aqueous media and is easily oxidized [30]. The presence of high concentrations of chloride ions, however, stabilizes the ion in the form of the highly soluble dichlorocuprous anion $\left(\mathrm{CuCl}_{2}\right)^{-}$ [25], [26], [31] through the reaction:

$$
\mathrm{Cu}^{+}+2 \mathrm{Cl}^{-} \rightarrow \mathrm{CuCl}_{2}^{-}
$$

Equation (4) may be rewritten in terms of a complexation equilibrium which relates the relative concentrations of the ionic species in solution [25], thus:

$$
\frac{\left[\mathrm{CuCl}_{2}^{-}\right]}{\left[\mathrm{Cu}^{+}\right]\left[\mathrm{Cl}^{-}\right]^{2}}=3.48 \times 10^{5}
$$

This reveals that in an electrolyte of $620 \mathrm{mM} \mathrm{Cl}^{-}$nearly $100 \%$ of the $\mathrm{Cu}^{+}$ion is complexed in the form of the dichlorocuprous anion since the ratio of $\left(\mathrm{CuCl}_{2}\right)^{-}$to $\mathrm{Cu}^{+}$is approximately $1.3 \times 10^{5}$. The dominance of this species of copper chloride complex at chloride concentrations between $10 \mathrm{mM}$ and $1 \mathrm{M}$ has been experimentally verified by others [32]. Hence, the second reduction reaction given by (3) is unlikely to occur as written in electrolytes with a high chloride concentration and therefore the reduction of $\mathrm{Cu}^{2+}$ more likely proceeds as an ECE process, thus:

$$
\begin{array}{lll}
\text { E } & \mathrm{Cu}^{2+}+\mathrm{e}^{-} \rightarrow \mathrm{Cu}^{+} & \text {(Peak 1) } \\
\mathrm{C} & \mathrm{Cu}^{+}+2 \mathrm{Cl}^{-} \rightarrow \mathrm{CuCl}_{2}^{-} & \text {(Peak 2) }
\end{array}
$$

During the reverse scan, there appears to be a difference in the processes governing the oxidation of metallic copper to $\mathrm{Cu}^{2+}$ depending on the $\mathrm{Cu}^{2+}$ concentration. Evidence for this includes the emergence of a double apex in the shape of the stripping peak in Fig. 4 and the greater than expected shift in the potential of Peak 4. However, it is proposed that changes in the $\mathrm{Cu}^{2+}$ concentration are not responsible for these observations per se, but rather the formation of a range of chlorocopper(I) complexes produced from adsorbed chloride at the working electrode surface and dependant on the concomitant change in $\mathrm{Cl}^{-}$concentration. In this situation we can consider the stripping of metallic copper and the oxidation of resultant products to the cupric ion as being described by successive single electron transfer processes at the electrode surface separated by a chemical reaction in solution as described by the generic reactions:

$$
\begin{array}{lll}
\text { E } & \mathrm{Cu}-\mathrm{e}^{-} \rightarrow \mathrm{Cu}^{+} & \text {(Peak 3) } \\
\mathrm{C} & \mathrm{Cu}^{+}+(\mathrm{n}+1) \mathrm{Cl}^{-} \rightarrow \mathrm{CuCl}_{(\mathrm{n}+1)}^{\mathrm{n}-} & \\
\mathrm{E} & \mathrm{CuCl}_{(\mathrm{n}+1)}^{\mathrm{n}-}-\mathrm{e}^{-} \rightarrow \mathrm{Cu}^{2+}+(\mathrm{n}+1) \mathrm{Cl}^{-} & (\text {Peak 4) }
\end{array}
$$

Likely values for " $n$ " in the equations above include: $n=0$, resulting in the formation of cuprous chloride, $\mathrm{CuCl} ; \mathrm{n}=1$, resulting in the formation of the dichlorocuprous anion, $\left(\mathrm{CuCl}_{2}\right)^{-}$; and $\mathrm{n}=2$, resulting in the formation of $\left(\mathrm{CuCl}_{3}\right)^{2-}$. The presence of these chlorocopper(I) complexes during the oxidation of $\mathrm{Cu}^{+}$in chloride media has been reported by others [30], [31] and from a theoretical analysis it has been shown that their relative proportions are dependant on the chloride concentration. For example, Xiao et al. calculated the speciation of chlorocopper(I) complexes as a function of temperature and total chloride concentration [32]. They report that at $25{ }^{\circ} \mathrm{C}$ the percentage of $\mathrm{Cu}^{+}$ions in the form of $\mathrm{CuCl}$ reaches a maximum of about $60 \%$ at a chloride concentration of approximately $1 \mathrm{mM}$ before dropping back to a level of about $5 \%$ as the chloride concentration is increased to $1 \mathrm{M}$. By contrast, the percentage of $\mathrm{Cu}^{+}$ions in the form of $\left(\mathrm{CuCl}_{2}\right)^{-}$is practically zero at chloride concentrations below $1 \mathrm{mM}$ but rises almost linearly to a peak value of about $85 \%$ at a chloride concentration of approximately $100 \mathrm{mM}$ before dropping back to about $75 \%$ as the chloride concentration is raised further toward $1 \mathrm{M}$. Similarly, they show that $\left(\mathrm{CuCl}_{3}\right)^{2-}$ is not present until the chloride concentration is of the order of $100 \mathrm{mM}$ and that the percentage of $\mathrm{Cu}^{+}$ions contained in this form rises with increasing chloride reaching a value of about $20 \%$ at $1 \mathrm{M}$ chloride.

In two of the experiments reported here, the chloride concentration ranged from $620 \mathrm{mM}$ to $810 \mathrm{mM}$ as the $\mathrm{Cu}^{2+}$ concentration was increased. At these levels of chloride we therefore expect stripped $\mathrm{Cu}^{+}$ions to complex with adsorbed chloride at the working electrode surface predominantly in the forms of $\left(\mathrm{CuCl}_{2}\right)^{-}$and $\left(\mathrm{CuCl}_{3}\right)^{2-}$ with the former initially at a higher concentration than the latter but with the ratio of former to latter decreasing as the chloride concentration increases (viz. $\mathrm{Cu}^{2+}$ concentration). This would then explain the changing shape of the stripping peak with $\mathrm{Cu}^{2+}$ concentration shown in Fig. 4: at low $\mathrm{Cu}^{2+}$ concentrations $\mathrm{Cu}^{+}$ is mostly stripped in the form of $\left(\mathrm{CuCl}_{2}\right)^{-}$but as the $\mathrm{Cu}^{2+}$ concentration increases an increasing proportion of $\mathrm{Cu}^{+}$is stripped in the form of $\left(\mathrm{CuCl}_{3}\right)^{2-}$ (at a more anodic potential) resulting in the emergence of a double current peak.

This also explains the shifting position of the potential for Peak 4 in Fig. 4. If the electrode potential given by reaction (11) is more positive for chlorocopper(I) complexes where $\mathrm{n}=$ 2 than for $\mathrm{n}=1$, then as the concentration of $\left(\mathrm{CuCl}_{3}\right)^{2-}$ increases whilst that for $\left(\mathrm{CuCl}_{2}\right)^{-}$decreases with rising $\mathrm{Cu}^{2+}$ levels, the resultant combination of the two superimposed peaks would shift toward more anodic potentials.

The data shown in Fig. 7 further clarifies the nature of the oxidation processes. The chloride concentration in the reduced potential scan range experiment also varied between $620 \mathrm{mM}$ and $810 \mathrm{mM}$, but here no significant shift in the potential of 


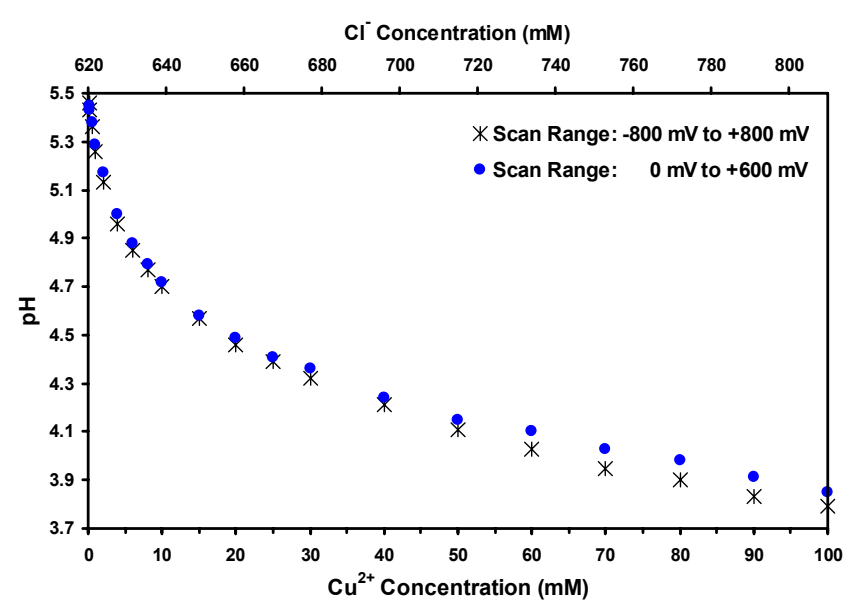

Fig.10. Variation in $\mathrm{pH}$ as a function of $\mathrm{Cu}^{2+}$ and $\mathrm{Cl}^{-}$concentrations.

the oxidation peak is observed. This suggests that the speciation of chlorocopper(I) complexes depends not only on the chloride concentration, but also on the source of $\mathrm{Cu}^{+}$ions: different complexes are only formed in significant quantities when adsorbed chloride reacts with $\mathrm{Cu}^{+}$ions stripped from metallic copper whereas complextion of chloride and $\mathrm{Cu}^{+}$ions in solution predominantly forms just the single $\left(\mathrm{CuCl}_{2}\right)^{-}$ species. This is not unreasonable since formation of more than one species of chlorocopper(I) complex requires a variable energy source, which can only be attained electronically at the electrode surface.

Fig. 10 shows the measured $\mathrm{pH}$ at each $\mathrm{Cu}^{2+}$ concentration for both experiments. This shows that over the $\mathrm{Cu}^{2+}$ range investigated, the solution becomes increasingly acidic dropping by nearly $2 \mathrm{pH}$ units. The data also shows that the change in $\mathrm{pH}$ is almost identical between the two experiments. This implies that observed differences in the $\mathrm{Cu}^{2+} / \mathrm{Cu}^{+}$redox couple response between the two experiments can not be attributed to $\mathrm{pH}$.

\section{B. Limits of Detection}

The concept of a limit of detection for a planar electrode operating as a voltammetric sensor is somewhat ambiguous. Whilst it is possible to determine a figure for the lowest concentration of a target analyte that can be detected by a given electrode in a controlled experiment, this value ultimately depends upon the physical size of the electrode, the sensitivity of the measurement equipment, the composition of the supporting electrolyte and the type of electroanalytical technique employed (e.g. cyclic, pulsed or stripping). Nevertheless, as an indicator of the potential capabilities of screen-printed electrodes, the data of Fig. 9 reveal that cupric ion concentrations as low as $10 \mu \mathrm{M}\left(\approx 0.6 \times 10^{-3} \mathrm{gL}^{-1}\right)$ will produce current peaks that are detectable above background noise levels in chloride media for an electrode with the relatively small area of approximately $4.0 \mathrm{~mm}^{2}$ when using differential pulse voltammetry. This is a more than adequate level of sensitivity in many corrosion monitoring applications including, for example, detection of the onset of crevice corrosion in nickel-aluminium bronze alloys where cupric ion concentrations as high as $80 \mathrm{mM}$ have been reported during the first month of corrosion [1].

\section{CONClusions}

Screen-printed platinum electrodes demonstrate a detectable response to cupric ions in a chloride background, though it is clear from the results that there are differences in the response between reduction and oxidation cycles, suggesting that the reaction mechanisms are not straightforward. We propose that in $3.5 \% \mathrm{NaCl}$ the reduction of $\mathrm{Cu}^{2+}$ to metallic copper proceeds as two successive single-electron reactions separated by an intermediate chemical step where the cuprous ion $\left(\mathrm{Cu}^{+}\right)$ is converted to the dichlorocuprous anion. By contrast, the oxidation of metallic copper back to the cupric ion $\left(\mathrm{Cu}^{2+}\right)$ depends on the chloride concentration and may involve the formation of several different chlorocopper(I) complexes resulting from complexation of stripped $\mathrm{Cu}^{+}$ions with chloride adsorbed at the electrode surface.

Because the oxidation of copper is strongly dependent on the chloride concentration, operation of this sensor over potential ranges that result in the plating of metallic copper on the electrode surface is not recommended. Instead, a reasonably linear cupric ion sensor can be attained by performing voltammetry over the potential range that encompasses the redox couple $\mathrm{Cu}^{2+} / \mathrm{Cu}^{+}$only. The results also indicate that the electrodes could be used in an amperometric mode where the detection of $\mathrm{Cu}^{2+}$ concentration is inferred from the measurement of the $\mathrm{Cu}^{2+}$ to $\mathrm{Cu}^{+}$reduction reaction. Here, the working electrode would be held at a fixed potential (as given by the value of Peak 1 which has been shown to remain invariant over $\mathrm{Cu}^{2+}$ concentrations up to $100 \mathrm{mM}$ ) and the measured current would be directly proportional to the concentration.

This investigation has also shown that screen-printed platinum electrodes demonstrate identical behavior to that of pure platinum under the same conditions, giving confidence in their use as a cheaper alternative electrode material. The typical processing cost for one of these screen-printed electrodes in a small batch size of around 100 devices works out at around less than $\$ 1$ per device, which is considerably cheaper than a comparable sized platinum metal electrode (e.g. the eDAQ $1 \mathrm{~mm}$ diameter platinum voltammetric disk electrode, EQ-ET075-1, at over \$200). Screen-printing technology also offers the possibility of integrating a number of different electrodes or electrochemical sensors onto a common platform to produce cheap sensor arrays that may be able to detect a range of chemical species within a small localized region. This would be advantageous in certain corrosion monitoring applications where location issues make the deployment of a number of conventional ion sensors extremely difficult. For example, the authors have already developed a screen-printed electrode for chloride detection with lifetime in excess of 1 year, as well as pH sensors and dissolved oxygen sensors with acceptable lifetimes of at least 230 days, demonstrating the robustness of screen-printing technology [33], [34]. Finally, their ability to operate in acidic 
chloride environments and remain intact makes them ideal sensors for marine based corrosion measurements.

\section{REFERENCES}

[1] J. A. Wharton, and K. R. Stokes, "Analysis of nickel-aluminium bronze crevice solution chemistry using capillary electrophoresis," Electrochem. Comms., vol. 9, pp. 1035-1040, May 2007.

[2] A. R. Firooz, M. Mazloum, J. Safari, and M. K. Amini, "Coated-wire copper(II)-selective electrode based on phenylglyoxal- $\alpha$-monoxime ionophore," Anal. Bioanal. Chem., vol. 372, pp. 718-722, Feb. 2002

[3] A. Sil, V. S. Ijeri, and A. K. Srivastava, "Coated wire copper(II) ion selective electrode based on 5,6,14,15-dibenzo-1,4-dioxa-8,12 diazacyclopentadeca-5,14-diene," Electroanalysis, vol. 14, pp. 12151217, Sept. 2002.

[4] D. Vlascici, E. F. Cosma, E. M. Pica, V. Cosma, O. Bizerea, G. Mihailescu, and L. Olenic, "Free base porphyrins as ionophores for heavy metal sensors," Sensors, vol. 8, pp. 4995-5004, Aug. 2008.

[5] M. R. Ganjali, M. Emami, and M. Salavati-Niasari, "Novel copper(II)selective sensor based on a new hexadentates Schiff's base," Bull. Korean Chem. Soc., vol. 23, pp. 1394-1398, June 2002.

[6] V. K. Gupta, R. Prasad, and A. Kumar, "Preparation of ethambutolcopper(II) complex and fabrication of PVC based membrane potentiometric sensor for copper," Talanta, vol. 60, pp. 149-160, Jan. 2003.

[7] V. K. Gupta, R. Prasad, and A. Kumar, "Cu(II) selective sensor based on $5,7,12,14$ tetramethyldibenzo[b,i]-1,4,8,11-tetraazacyclotetradecane in PVC matrix", J. Applied Electrochemistry, vol. 33, pp. 381-386, Jan. 2003.

[8] V. K. Gupta, A. K. Jain, G. Maheshwari, H. Lang, and Z. Ishtaiwi, "Copper(II)-selective potentiometric sensors based on porphyrins in PVC matrix," Sensors and Actuators B, vol. 117, pp. 99-106, Sept (2006).

[9] R. Koncki, Ł. Tymecki, E. Zwierkowska, and S. Głab, "Screen-printed copper ion-selective electrodes," Fresenius J. Anal. Chem., vol. 367, pp 393-395, June 2000.

[10] Ł. Tymecki, M. Jakubowska, S. Achmatowicz, R. Koncki, and S. Głab, "Potentiometric thick-film graphite electrodes with improved response to copper ions," Analytical Letters, vol. 341, pp. 71-78, Jan. 2001.

[11] A. Abbaspour, and S. M. M. Moosavi, "Chemically modified carbon paste electrode for determination of copper(II) by potentiometric method," Talanta, vol. 56, pp. 91-96, Jan. 2002.

[12] G. B. Oglesby, W. C. Duer, and F. J. Millero, "Effect of chloride ion and ionic strength on the response of a copper(II) ion-selective electrode," Anal. Chem., vol. 49, pp. 877-879, May 1977.

[13] K. Y. Chumbimuni-Torres, P. Calvo-Marzal, J. Wang, and E. Bakker, "Electrochemical sample matrix elimination for trace-level potentiometric detection with polymeric membrane ion-selective electrodes," Anal. Chem., vol. 80, pp. 6114-6118, June 2008.

[14] C. S. Brossia, and R. G. Kelly, "Occluded solution chemistry control and the role of alloy sulphur on the initiation of crevice corrosion in type 304SS," Corrosion Science, vol. 40, pp. 1851-1871, Nov. 1998.

[15] S. P. White, G. J. Weir, and N. J. Laycock, "Calculating chemical concentrations during the initiation of crevice corrosion," Corrosion Science, vol. 42, pp. 605-629, Apr. (2000).

[16] J. A. Wharton, and K. R. Stokes, "The influence of nickel-aluminium bronze microstructure and crevice solution on the initiation of crevice corrosion," Electrochimica Acta, vol. 53, pp. 2463-2473, Jan. 2008.

[17] G. F. Kennell, R. W. Evitts, and K. L. Heppner, "A critical crevice solution and IR drop crevice corrosion model," Corrosion Science, vol. 50, pp. 1716-1725, June 2008.

[18] K. C. Honeychurch, D. M. Hawkins, J. P. Hart, and D. C. Cowell, "Voltammetric behaviour and trace determination of copper at a mercury-free screen-printed carbon electrode," Talanta, vol. 57, pp. 565-574, May 2002

[19] R. S. Freire, and L. T. Kubota, "Application of self-assembled monolayer-based electrode for voltammetric determination of copper," Electrochimica Acta, vol. 49, pp. 3795-3800, Sep. 2004

[20] E. R. de Sousa, E. P. Marques, E. N. Fernandes, J. Zhang, and A. L. B. Marques, "Graphite electrodes modified by 8 -Hydroxyquinolines and its application for the determination of copper in trace levels," J. Braz. Chem. Soc., vol. 17, pp. 177-183, Jan. 2006.
[21] A. Rahafrooz and S. Pourkamali, "Detection of sub-ppm traces of aqueous heavy-metal ions using micro-electro-mechanical beam resonators," J. Micromech. Microeng., vol. 19, 115003,. Nov. 2009.

[22] F. J. Millero, "The physical chemistry of seawater," Annual Review of Earth and Planetary Sciences, vol. 2, pp. 101-150, May 1974.

[23] A. Molina, and I. Morales, "Comparison between derivative and differential pulse voltammetric curves of EC, CE and catalytic processes at spherical electrodes and microelectrodes," Int. J. Electrochem. Sci., vol. 2, pp. 386-405, May 2007.

[24] S. H. Cadle, and S. Bruckenstein, "Rotating ring disk study of the underpotential deposition of copper at platinum in $0.5 \mathrm{M}$ hydrochloric acid," Analytical Chemistry, vol. 43, pp. 932-933, June 1971.

[25] N. M. Marković, H. A. Gasteiger, and P. N. Ross Jr, "Copper electrodeposition on Pt(111) in the presence of chloride and (bi)sulphate: Rotating ring - Pt(111) disk electrode studies," Langmuir, vol. 11. pp. 4098-4108, Oct. 1995.

[26] C. P. de Leon, and F. C. Walsh, "Research and development techniques 1: Potentiodynamic studies of copper metal deposition," Trans. Int. Met. Fin., vol. 81, pp. B95-B100, Sep. 2003.

[27] A. Habib, T. Shireen, A. Islam, N. Begum, and A. M. S Alam, "Cyclic voltammetric studies of copper and manganese in the presence of Lleucine using glassy carbon electrode," Pak. J. Anal. \& Envir. Chem., vol. 7, pp. 96-102, 2006.

[28] L. Trnkova, L. Zerzankova, F. Dycka, R. Mikelova, and F. Jelen, "Study of copper and purine-copper complexes on modified carbon electrodes by cyclic and elimination voltammetry," Sensors, vol. 8, pp. 429-444, Jan. 2008.

[29] S. C. Rifkin, and D. H. Evans, "Analytical evaluation of differential pulse voltammetry at stationary electrodes using computer-based instrumentation," Anal. Chem., vol. 48, pp. 2174-2179, Dec. 1976.

[30] M. Wang, Y. Zhang, and M. Muhammed, "Critical evaluation of thermodynamics of complex formation of metal ions in aqueous solutions III. The system $\mathrm{Cu}(\mathrm{I}, \mathrm{II})-\mathrm{Cl}^{-}-\mathrm{e}$ at $298.15 \mathrm{~K}$," Hydrometallurgy, vol. 45, pp. 53-72, May 1997.

[31] G. Kear, B. D. Barker, and F. C. Walsh, "Electrochemical corrosion of unalloyed copper in chloride media - a critical review," Corrosion Science, vol. 46, pp. 109-135, Jan. 2004.

[32] Z. Xiao, C. H. Gammons, and A. E. Williams-Jones, "Experimental study of copper(I) chloride complexing in hydrothermal solutions at 40 to $300^{\circ} \mathrm{C}$ and saturated water vapour pressure," Geochimica et Cosmochimica Acta, vol. 62, pp. 2949-2964, Sep. 1998.

[33] A. Cranny, N. R. Harris, M. Nie, J. A. Wharton, R. J. K. Wood, and K. R. Stokes, "Screen-printed potentiometric $\mathrm{Ag} / \mathrm{AgCl}$ chloride sensors: Lifetime performance and their use in soil salt measurements," Sensors and Actuators A: Physical, vol. 169, pp. 288-294, Oct. 2011.

[34] J. K. Atkinson, A. W. J. Cranny, W. V. Glasspool, and J. A. Mihell, “An investigation of the performance characteristics and operational lifetimes of multi-element thick film sensor arrays used in the determination of water quality parameters," Sensors and Actuators B: Chemical, vol. 54, pp. 215-231, March. 1999. 